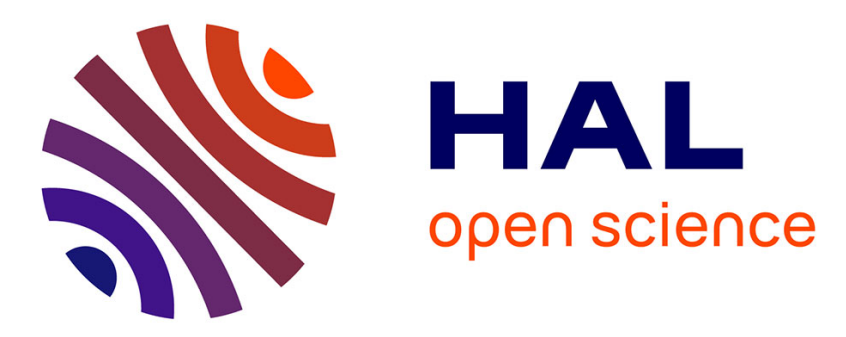

\title{
Anisotropy dynamics of CuMn spin glass through torque measurements
}

\author{
J.B. Pastora, T.W. Adair, D.P. Love
}

\section{To cite this version:}

J.B. Pastora, T.W. Adair, D.P. Love. Anisotropy dynamics of $\mathrm{CuMn}$ spin glass through torque measurements. Journal de Physique Lettres, 1983, 44 (20), pp.859-863. 10.1051/jphyslet:019830044020085900 . jpa-00232274

\section{HAL Id: jpa-00232274 https://hal.science/jpa-00232274}

Submitted on 1 Jan 1983

HAL is a multi-disciplinary open access archive for the deposit and dissemination of scientific research documents, whether they are published or not. The documents may come from teaching and research institutions in France or abroad, or from public or private research centers.
L'archive ouverte pluridisciplinaire HAL, est destinée au dépôt et à la diffusion de documents scientifiques de niveau recherche, publiés ou non, émanant des établissements d'enseignement et de recherche français ou étrangers, des laboratoires publics ou privés. 
Classification

Physics Abstracts

$75.30 \mathrm{G}-75.40$

\title{
Anisotropy dynamics of CuMn spin glass through torque measurements
}

\author{
J. B. Pastora, T. W. Adair III and D. P. Love \\ Physics Department, Texas A \& M University, College Station, Texas 77843, U.S.A.
}

(Reçu le 6 juin 1983, révisé le 19 août, accepté le 29 août 1983)

\begin{abstract}
Résumé. - La dynamique de l'énergie d'anisotropie, $E_{\mathrm{a}}$, est étudiée sur un échantillon de verre de spin $\mathrm{CuMn}$ : 0,5 at. \% par mesure de couple. Après réduction à zéro du champ de refroidissement dirigé suivant l'axe des $\hat{Z}$, et après un délai suffisant pour rendre négligeable l'effet du déclin temporel de l'aimantation, un champ de mesure $H$ est appliqué à un angle de $90^{\circ}$ par rapport à l'axe des $\hat{Z}$. Le couple d'anisotropie $\Gamma$ décroît par suite de la redéfinition de la direction $\theta_{N}$ de l'axe d'anisotropie $\hat{N}$ par rapport à l'axe des $\hat{Z}$ pour $H \geqslant 70$ Oe et $T=2,39 \mathrm{~K}$. L'angle $\theta_{N}$ croît avec $H$ et $T$. $E_{\mathrm{a}}$ s'avère être unidirectionnelle seulement.
\end{abstract}

\begin{abstract}
The dynamics of anisotropy energy, $\left(E_{\mathrm{a}}\right)$, in a CuMn $0.5 \%$ spin glass sample is investigated by torque measurements. After reducing the cooling field along the $\hat{Z}$ axis to zero and delaying to allow the time decay of magnetization to diminish, a measuring field $H$ is applied $90^{\circ}$ from $\hat{Z}$. The anisotropy torque $\Gamma$ decays due to redefinition of the direction $\theta_{N}$ of the anisotropy axis $\hat{N}$ with respect to $\hat{Z}$ for $H \geqslant 70$ Oe at $T=2.39 \mathrm{~K}$. The angle $\theta_{N}$ increases with $H$ and $T$. $E_{\mathrm{a}}$ is found to be unidirectional only.
\end{abstract}

The macroscopic anisotropy energy of metallic spin glasses has been investigated through magnetization [1, 2], susceptibility $[3,4]$, ESR [5, 6], NMR [7] and torque $[4,8,9]$ measurements. The usual theoretical approach $[3,5,10,11]$ is to assume that the remanent magnetization $\sigma$ undergoes rigid rotations about an anisotropy axis $\hat{N}$ which remains fixed, although Saslow has developed (following a suggestion of Monod) a theory which permits the anisotropy to be redefined by the measurement [12]. An anisotropy energy term, $E_{\mathrm{a}}(\theta)$ is usually introduced into the macroscopic free energy $E$, such that with a measuring magnetic field $\mathbf{H}$,

$$
E=E_{\mathrm{a}}(\theta)-\boldsymbol{\sigma} . \mathbf{H}
$$

where $\theta$ is the angle between $\sigma$ and $\hat{N}$. It has been established [2] that for $\operatorname{CuMn}, E_{\mathrm{a}}(\theta)$ has a strong unidirectional ( $2 \pi$-periodic) component. Iwata et al. [8] also claimed to have measured a weak uniaxial $(\pi$-periodic) component. Since then, many experiments $[3,5,13]$ have been analysed considering both types of contributions to $E_{\mathrm{a}}(\theta)$, i.e. :

$$
E_{\mathrm{a}}(\theta)=-K_{1} \cos \theta+K_{2} \sin ^{2} \theta
$$

where $K_{1}$ and $K_{2}$ are the unidirectional and uniaxial anisotropy constants respectively. Recent experimental evidence however [4,9], points to the fact that for CuMn only the unidirectional component is present. 
Under equilibrium conditions, equation 1 yields

$$
\frac{\partial E}{\partial \theta}=0=\frac{\mathrm{d} E_{\mathrm{a}}(\theta)}{\mathrm{d} \theta}-\sigma H \sin \left(\theta_{H}-\theta\right)
$$

where $\theta_{H}$ is the angle between $\mathbf{H}$ and $\hat{N}$. That is, the anisotropy torque $\Gamma_{\mathrm{a}}$ (which is equal and opposite to the measured Zeeman torque) acting on $\sigma$ is given by :

$$
\Gamma_{\mathrm{a}}=\frac{\mathrm{d} E_{\mathrm{a}}(\theta)}{\mathrm{d} \theta}=K_{1} \sin \theta+K_{2} \sin 2 \theta
$$

assuming both types of contributions to $E_{\mathrm{a}}(\theta)$ as in (2).

In this letter we report anisotropy torque decay measurements on a CuMn $0.5 \%$ sample, which show a redefinition of the anisotropy axis $\hat{N}$ by the measuring field $\mathbf{H}$, even for relatively small values of $\mathbf{H}(\geqslant 70 \mathrm{Oe})$. Our results also indicate the presence of only an unidirectional component of the anisotropy energy.

Our measurements are made with a compensating torque magnetometer to be described elsewhere [14]. The experimental situation is shown in figure 1. The sample is cooled through the spin glass temperature, $T_{0}=8 \mathrm{~K}$, in the presence of a $7 \mathrm{kOe}$ magnetizing field along the $\hat{Z}$ axis. This field causes a remanent magnetization $\sigma_{0}$ and establishes the initial direction $\hat{N}_{0}$ of the anisotropy axis, which coincides with $\hat{Z}$. It is a well known fact [15] that $\sigma_{0}$ decays with time, thus after reducing the cooling field to zero, a time delay of $85 \pm 5 \mathrm{~min}$. is allowed to prevent $\sigma$ decay from being an important factor in our measurements. Then a small measuring field $\mathbf{H}$ is applied along $\hat{Z}$. Under these conditions, the torque on the sample is zero. Then $\mathbf{H}$ is rapidly rotated to a direction $90^{\circ}$ from $\hat{Z}$. The torque $\Gamma$ has a maximum value when $\mathbf{H}$ reaches the $90^{\circ}$ position and then starts to decrease very rapidly. (Actually $\Gamma$ was found to decay at all angles $\geqslant 10^{\circ}$ for $H=700$ Oe but we decided to do the study by systematically rotating $H$ to $90^{\circ}$ from $\hat{Z}$ in order to maximize the effect for small fields). Typical measurements of the decay part of the cycle after $\Gamma$ has reached

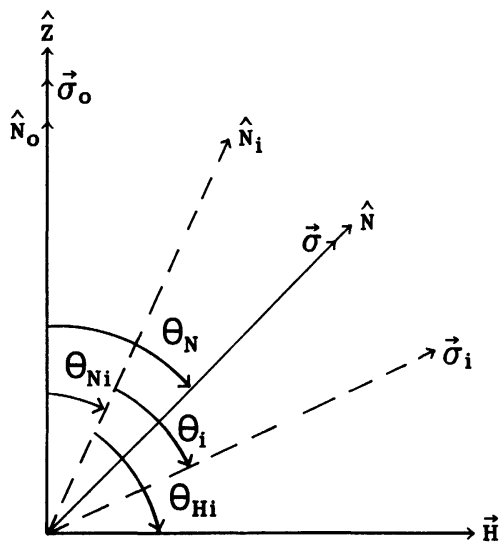

Fig. 1. - Experimental conditions for torque decay measurements. The sample is cooled through the spin glass temperature in a $7 \mathrm{kOe}$ field $H_{\mathrm{c}}$ along $\hat{Z}$. This induces a remanent magnetization $\sigma_{0}$ and anisotropy axis $\hat{N}_{0}$. We delay for over an hour after reducing $H_{\mathrm{c}}$ to zero to allow $\sigma$ to decay. Then a small $\mathbf{H}$ is set along $\hat{Z}$. $\mathrm{H}$ is rotated $(\sim 8 \mathrm{~s})$ to $90^{\circ}$ away from $\hat{Z}, \Gamma$ increases to a maximum and then it starts to decay when $H$ is at $90^{\circ}$. After a $\Gamma$ decay curve is completed, $\mathbf{H}$ is decreased and rotated back toward $\hat{Z}$. A direction $\theta_{N}$ is found for which $\Gamma=0$. Further rotation of the magnet toward $\hat{Z}$ yields a torque of opposite sign. $\theta_{N}$ is the direction $\hat{N}$ has been redefined to by the measurement. The dashed lines indicate the position of $\hat{N}$ and $\sigma$ sometime during $\Gamma$ decay. 
a maximum value are shown in figure 2 as a function of measuring field and of temperature. After data for a decay curve has been obtained, $H$ is reduced to a small value $(\sim 10 \mathrm{Oe})$ in order to prevent any further distortion of the system. Then the magnet is rotated back towards $\dot{Z}$ (Fig. 1), which causes $\Gamma$ to decrease to zero at. a new direction $\theta_{N}$. Upon further rotation of the magnet toward $\dot{Z}$, a torque of the opposite sign will appear. The only possibility for $\Gamma$ to be zero at $\theta_{N}$ is for $\sigma$ to coincide with $\hat{N}$ at that angle. These results show that the anisotropy axis has been redefined by the measuring field to a new direction $\theta_{N}^{0}$ away from $\hat{Z}$, a fact reported for the first time. Results of $\theta_{N} v s . H$ and $\theta_{N} v s . T$ are presented in figure 3 . There it is seen that redefinition of $\theta_{N}$ is most easily obtained at high $H$ and high $T$.

Redefinition of $\theta_{N}$ causes $\Gamma$ to decay in time due to the fact that the angle $\left(\theta_{H}-\theta\right)$ between $\sigma$ and $\mathbf{H}$ decreases. The dynamics of $\Gamma$ decay itself will be discussed elsewhere [14]. Preliminary results indicate the value of the anisotropy constant remains approximately the same after redefinition of $\theta_{N}$. This point will be more carefully investigated, but the redefinition of $\theta_{N}$ is the most important reason for the $\Gamma$ decay.

Our results of redefinition of the direction of net anisotropy $\left(\theta_{N}\right)$ by the measuring field are contrary to the usual assumption of rigid rotations of the remanent magnetization $\sigma$ around a fixed anisotropy axis, $\hat{N}$, frequently used to interpret most studies of anisotropy energy through different types of measurements. Also our results explain the irreversibility and time dependent effects found on previous ESR [13], torque [4, 9] and transverse susceptibility [3, 4] measurements at large values of $\theta_{H}$ and of measuring field $\mathbf{H}$; i.e., the net anisotropy direction is redefined by the measuring field, inducing irreversibility and time decay.

An extrapolation of the low field data of figure 3 shows (for the conditions of that figure) the existence of a threshold field $H_{\text {th }} \simeq 50$ Oe for which $\theta_{N}$ is zero. That is at $T=2.39 \mathrm{~K}$ and $H \leqslant$ $50 \mathrm{Oe}$ there is no redefinition of the anisotropy axis' direction. This $H_{\mathrm{th}}$ should decrease as tempe-
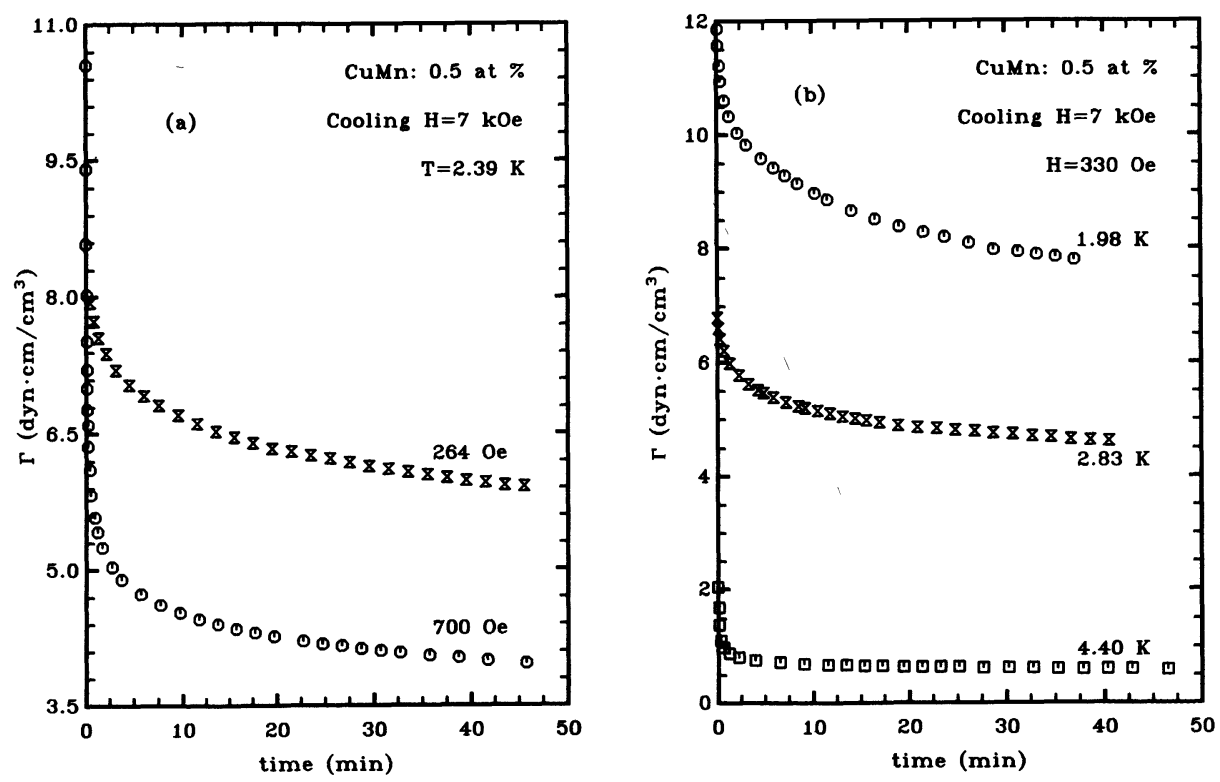

Fig. 2. - Typical torque decay curves for (a) constant temperature and (b) constant measuring field $\mathbf{H}$. At small values of $H, \Gamma$ decays gradually while at larger $H(700 \mathrm{Oe})$ about $90 \%$ of the total decay occurs during the first four minutes. That means redefinition of anisotropy axis direction occurs very rapidly at large $H$. Similarly, at high $T(4.4 \mathrm{~K})$ most of the decay occurs in the first couple of minutes. Thus the anisotropy direction is most easily redefined at high $H$ and high $T$. For this sample $T_{0} \simeq 8 \mathrm{~K}$. 


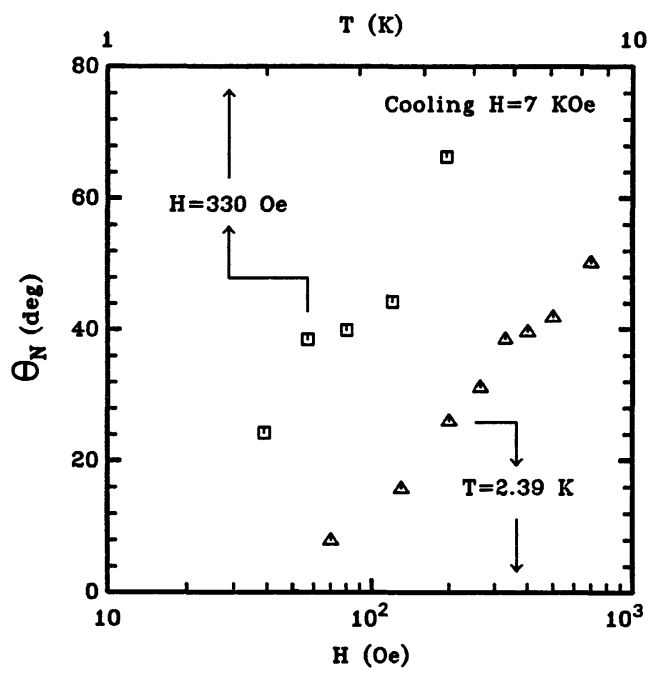

Fig. 3. - Variation of redefinition of anisotropy direction, $\theta_{N}$ with measuring field $H$ and temperature $T$. A given data point is taken after $45 \mathrm{~min}$. of corresponding $\Gamma$ decay. $\theta_{N}$ is most easily redefined at high $T$ and large $H$. Extrapolation of $\theta_{N} v s$. $H$ indicates the existence of a threshold field $H_{\text {th }} \simeq 50 \mathrm{Oe}$ for which $\theta_{N}=0$. Thus, for our sample at $T=2.39 \mathrm{~K}$, torque curves $\left(\Gamma v s . \theta_{H}\right)$ should be measured with $H<50 \mathrm{Oe}$ to avoid torque decay due to redefinition of $\theta_{N}$.

rature increases, and vice versa, as indicated by the larger net decay of the $4.4 \mathrm{~K}$ curve in figure $2 \mathrm{~b}$. Thus any study of anisotropy energy through any type of measurement (ESR, $\chi_{\perp}$, etc.) should be done with measuring fields lower than the threshold field corresponding to the given conditions of temperature and impurity concentration, in order to avoid redefinition of the anisotropy axis' direction.

We performed measurements of $\Gamma$ vs. $\theta_{H}$ for $T=2.39 \mathrm{~K}$ and $H<50 \mathrm{Oe}$. The $\Gamma$ vs. $\theta_{H}$ curves obtained are $2 \pi$-periodic. These results indicate that $E_{\mathrm{a}}(\theta)$ is unidirectional only. Otherwise a uniaxial ( $\pi$-periodic) shape contribution should have appeared at small $H$ before redefinition of anisotropy direction occurs. The anisotropy energy also stays essentially unidirectional after $\theta_{N}$ has been redefined by a large measuring field.

We have reported here results for a CuMn $0.5 \%$ spin glass sample which for the experimental conditions of figure 1 demonstrate that after removing the time decay of $\sigma$, for measuring fields $>50 \mathrm{Oe}$ the anisotropy axis $\hat{N}$ is still in a dynamic state. A redefinition of the orientation $\theta_{N}$ with respect to $\hat{Z}$ by the measuring field $\mathbf{H}$ in time is demonstrated. This effect is contrary to the assumption of rigid rotations of $\sigma$ around a fixed anisotropy axis commonly used to interpret most previous susceptibility, ESR, and torque measurements. This redefinition of $\theta_{N}$ together with $\Gamma$ vs. $\theta_{H}$ measurements at $H<50$ Oe show the anisotropy energy to be unidirectional only, in agreement with other workers $[4,9]$. Our results demonstrate that whenever a large measuring field applied away from $\hat{Z}$ is used for any experiment on this type of material, the system is being forced into a perturbed state, namely one with a new direction of the net anisotropy axis. In this situation one would not measure the intrinsic properties of the system, but the properties of the state the system is forced into by the measuring field.

\section{Acknow̧ledgments.}

We wish to thank Dr. W. M. Saslow for valuable conversations, Dr. P. Monod for providing the sample, and Dr. H. Armbruster and Dr. P. Colter for valuable discussions and technical advice. This research was supported by the Robert A. Welch Foundation. 


\section{References}

[1] Monod, P., Présean, J. J. and Tissier, B., J. Appl. Phys. 50 (1979) 7234.

[2] Koúvel, J. S., J. Phys. Chem. Solids 21 (1961) 57.

[3] Alloul, H. and Hippert, F., J. Physique Lett. 41 (1980) L-201.

[4] Hippert, F., Alloul, H. and Fert, A., in Proceedings of the Twenty-Eighth Conference on Magnetism and Magnetic Materials, Montreal (1982).

[5] Schultz, S., Gullikson, E. M., Fredkin, D. R. and Tovar, M., Phys. Rev. Lett. 45 (1980) 1508.

[6] Monod, P. and Berthier, Y., J. Magn. Magn. Mat. 15-18 (1980) 149.

[7] Alloul, H., J. Appl. Phys. 50 (1979) 7330.

[8] Iwata, T., Kai, K., Nakamichi, T. and Yamamoto, M., J. Phys. Soc. Jpn. 28 (1970) 582.

[9] Fert, A. and Hippert, F., Phys. Rev. Lett. 49 (1982) 1508.

[10] Saslow, W. M., Phys. Rev. B 22 (1980) 1174.

[11] FerT, A. and Levy, P. M., Phys. Rev. Lett. 44 (1980) 1538.

[12] Saslow, W. M., Phys. Rev. Lett. 48 (1982) 505.

[13] Gullikson, E. M., Fredkin, D. R. and Schultz, S., Phys. Rev. Lett. 50 (1983) 537.

[14] Pastora, J. B. and Adair, T. W., to be published.

[15] Présean, J. J., J. Physique Colloq. 39 (1978) C6-907. 